\title{
User Profiling in the Time of HTTPS
}

\author{
Roberto Gonzalez \\ NEC Labs. Europe \\ first.last@neclab.eu
}

\author{
Claudio Soriente \\ Telefonica Research \\ first.last@telefonica.com
}

\author{
Nikolaos Laoutaris \\ Telefonica Research \\ first.last@telefonica.com
}

\begin{abstract}
Tracking users within and across websites is the base for profiling their interests, demographic types, and other information that can be monetised through targeted advertising and big data analytics. The advent of HTTPS was supposed to make profiling harder for anyone beyond the communicating end-points. In this paper we examine to what extent the above is true. We first show that by knowing the domain that a user visits, either through the Server Name Indication of the TLS protocol or through DNS, an eavesdropper can already derive basic profiling information, especially for domains whose content is homogeneous. For domains carrying a variety of categories that depend on the particular page that a user visits, e.g., news portals, e-commerce sites, etc., the basic profiling technique fails. Still, accurate profiling remains possible through traffic fingerprinting that uses network traffic signatures to infer the exact page that a user is browsing, even under HTTPS. We demonstrate that transport-layer fingerprinting remains robust and scalable despite hurdles such as caching, dynamic content for different device types etc. Overall our results indicate that although HTTPS makes profiling more difficult, it does not eradicate it by any means.
\end{abstract}

\section{INTRODUCTION}

Online user profiling is a profitable business extensively carried out by third parties such as search engines, ad networks and network providers. It leverages browsing activities to infer user interests and intentions. Since HTTP traffic has no privacy provisions, any third party can pry on the connections

Permission to make digital or hard copies of all or part of this work for personal or classroom use is granted without fee provided that copies are not made or distributed for profit or commercial advantage and that copies bear this notice and the full citation on the first page. Copyrights for components of this work owned by others than ACM must be honored. Abstracting with credit is permitted. To copy otherwise, or republish, to post on servers or to redistribute to lists, requires prior specific permission and/or a fee. Request permissions from permissions@acm.org.

IMC 2016, November 14-16, 2016, Santa Monica, CA, USA

(c) 2016 ACM. ISBN 978-1-4503-4526-2/16/11 ..\$15.00

DOI: http://dx.doi.org/10.1145/2987443.2987451 to a websever and profile users. HTTPS enhances online user privacy by encrypting the communication between a browser and a webserver. Major internet stakeholders are pushing for an HTTPS everywhere web with the promise of increased security and privacy and, therefore, of mitigating the problem of user profiling by third parties.

In this paper we assess the extent by which HTTPS prevents third parties from profiling users based on the websites they visit. We show that the widely used Server Name Indication (SNI) extension of the TLS protocol leaks user interests to third parties which eavesdrop on the (encrypted) connection between a client and an HTTPS webserver. The SNI extension improves address-space utilization as it allows to consolidate several HTTPS webservers at a given IP address. However, SNI also hinders user privacy as it leaks the domain requested by a user, despite the HTTPS pledge of a secure and private connection.

The privacy leakage due to SNI is especially severe in websites with homogenous content across its pages. For example, a connection request to www. foxsports.com/tells a lot about the user interests, regardless of the actual page the user is browsing within that website. For a website with more variety across its pages, the domain requested by a user may not tell enough about the interests of that user. For example, a connection to www.amazon.com/ may not tell much about a user. However a connection to www.amazon.com/books/ would reveal interests in books and a connection to www.amazon.com/baby/ may indicate an intent to buy baby items. We show that by using traffic fingerprinting techniques, a network eavesdropper can accurately tell the page a user is browsing within a domain and, therefore, build a refined user profile.

Users profiling despite HTTPS is achievable, given enough bandwidth to fingerprint the websites under observation. In case bandwidth is an issue, we also define an optimization problem that allows an eavesdropper to periodically pick the websites to fingerprint in order to maximize the number of users that are accurately profiled over time. 
Overall, our findings show that HTTPS, while being a formidable tool to strengthen the security of web applications, cannot protect users against online profiling by third parties.

\section{BACKGROUND AND MODEL}

\section{User profiling.}

Profiling systems often use a closed-source mapping between URLs and interest categories. We follow the approach of previous work [1] and instantiate the mapping using the Display Planner of Google AdWords 2] - an online tool that given a URL returns the set of categories assigned by AdWords to that URL. Categories are arranged in a hierarchy and each URL has, on average, 10 assigned categories. The Display Planner also provides the inverse mapping, i.e., given a category it provides a list of websites that belong to that category.

\section{HTTPS and Server Name Indication.}

HTTPS enhances HTTP with the Transport Layer Security (TLS) protocol. TLS provides a secure pipe to a server that is usually authenticated via an X.509 certificate. The secure pipe is established via a TLS handshake - a procedure that allows the client and the server to establish cryptographic keys to encrypt and authenticate data exchanged through the pipe.

Given the ever increasing awareness on the privacy issues of HTTP, major web stakeholders are mandating secure (i.e., HTTPS) connections to serve their websites 3, 4]. Furthermore the Tor Project and EFF promote the HTTPS Everywhere extension [5, 6], that automatically redirects browsers to the HTTPS version of a website when available. One goal of this collective effort towards an HTTPS web is to increase online privacy with respect to network eavesdroppers. HTTPS ensures that a user is connected to the legitimate webserver and that the exchanged information cannot be eavesdropped by third parties 1

Server Name Indication (SNI) is an extension of the TLS protocol by which a client specifies the hostname it is attempting to connect in the client_hello message (the first message of a TLS handshake). The extension is widely used by modern browsers and allows a server to serve multiple HTTPS websites, each with its own X.509 certificate, from the same IP address. The SNI is, therefore, sent in cleartext and can be eavesdropped by any party tapping on the network between the client and the server.

\section{System Model.}

We consider a network eavesdropper that tries to profile users by tapping on their network connections.

\footnotetext{
${ }^{1}$ Security guarantees of HTTPS do not take into account phishing attacks or flaws in the public key certification system.
}

We assume an HTTPS everywhere web where users connect to any website via HTTPS. The network eavesdropper, therefore, does not have access to the cleartext traffic exchanged between the user browser and the webservers but only sees encrypted flows. However, we assume the eavesdropper can infer the hostname requested by the user by looking at the SNI in the client_hello message. In case SNI is not used, client queries to DNS (recall that DNS has no provisions for confidentiality) or simply a whois on the destination IP address may reveal the hostname requested by the user.

We simplify the structure of a website and the user browsing behaviour as follows. Each website has a main page and a set of 1-st level pages (i.e., the pages linked on the main page). We do not consider pages of the website beyond the ones linked on the main page, but our results can be easily generalized to account for more complex website structures. Similar to previous work $7,8,9,10,11$, we assume a user visits one page at a time for each domain 2 This could be either the main page, or any of the 1-st level pages. The eavesdropper tries to infer the page visited by the user and assigns to her profile the corresponding set of categories according to Google AdWords.

\section{USER PROFILING BY SNI}

Looking at the SNI in the client_hello message, a basic eavesdropper learns the website a user is browsing and assigns the categories of the main page to the user profile. If the user were actually browsing a page different from the main one, the profile built by the basic eavesdropper may not be accurate.

A first step towards understanding the accuracy of user profiling in an HTTPS everywhere web consists in assessing the difference between the categories of a website main page (e.g., the categories of www. nbcnews.com/) and the ones of any of its 1-st level pages (e.g, the categories of www.nbcnews.com/politics/).

In this experiment we have collected the list of top websites returned by AdWords for each of its 24 first level categories. Within each list, we have selected the 100 most popular websites based on their rank in Alexa 12]. For each of the resulting $2.4 \mathrm{~K}$ websites we have fetched the URL of all the links available on the main page that remains within the same host. We did not consider external links like the ones to CDNs. Each of the collected URLs (totalling to more than 110K URLs) was submitted to the AdWords Display Planner to obtain its set of categories.

For each of the 24 top level categories of AdWords, Figure 1 shows the distribution of the Jaccard index among the categories assigned to the main page of a website and the categories assigned to its 1-st level

\footnotetext{
${ }^{2}$ Discerning traffic when multiple pages of a domain are fetched simultaneously using HTTPS, remains an open problem.
} 


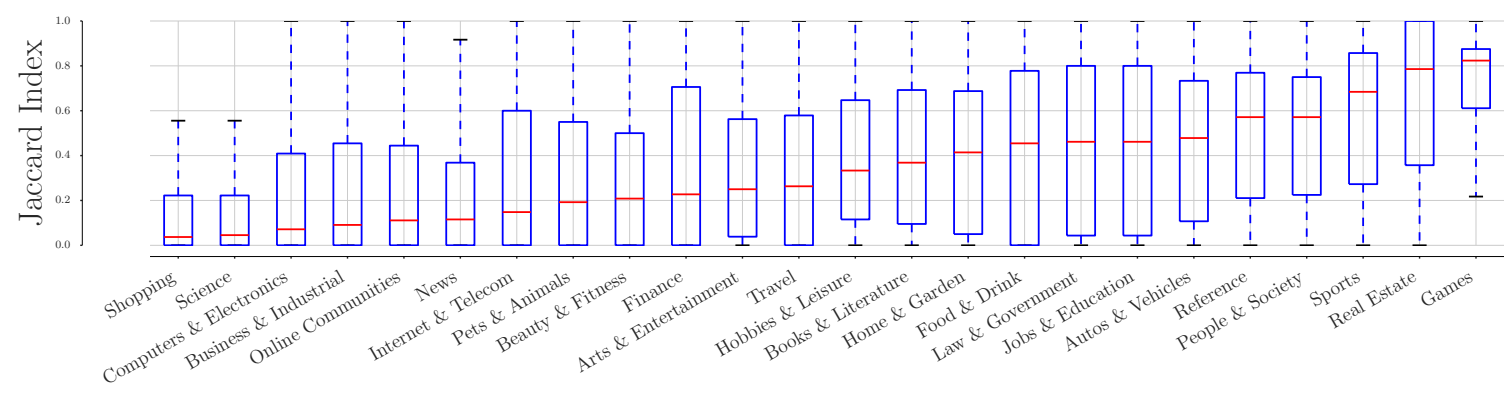

Figure 1: Distribution of the Jaccard index among the categories of the main page and the 1st-level pages.

pages. A Jaccard index close to 1 means that simply assigning the categories of the main page to a user creates a quite accurate profile, regardless of the actual page the user is browsing within that website. A Jaccard index close to 0 means that the same profile technique may lead to a less accurate user profile.

Figure 1 shows a great variance depending on the main category of the website. Users visiting Sports, Real Estate or Games websites could be profiled very accurately only by knowing the website their are connected to. However, when a user visits any page within a website related to, e.g., Shopping, Computers \& Electronics or News, the user profile built by assigning her the categories of the main page is likely to be inaccurate.

\section{TRAFFIC FINGERPRINTING}

In this section we show how to improve profiling accuracy by inferring the exact page a user is browsing using traffic fingerprinting. Traffic fingerprinting 7 , 8, 9, 10 is an active research area on techniques to infer information (such as the visited page on an encrypted connection) by solely observing traffic patterns at the network/transport level.

Fingerprinting involves a training phase during which the adversary builds a fingerprint of each of the monitored pages. This is accomplished by fetching multiple times the monitored pages and recording features of the generated traffic such as packet size or interarrival times. Later, the adversary eavesdrops on the client's connection, extracts the same features from the client's traffic, and tries to match the client trace to one of the fingerprints computed during the training phase. Differences between the training data and the client (or test) data, due to, e.g., different routes or congestions are mitigated using statistical methods.

We use and adapt to our scenario the fingerprinting technique of 11 - the most accurate web fingerprinting framework to date - that uses as features the size and the direction of each packet of a TCP connection. The classifier is, therefore, robust against differences in bandwidth or congestions along the route. The authors of [11 show that page fingerprinting is hard in an open-world scenario in which the client can browse

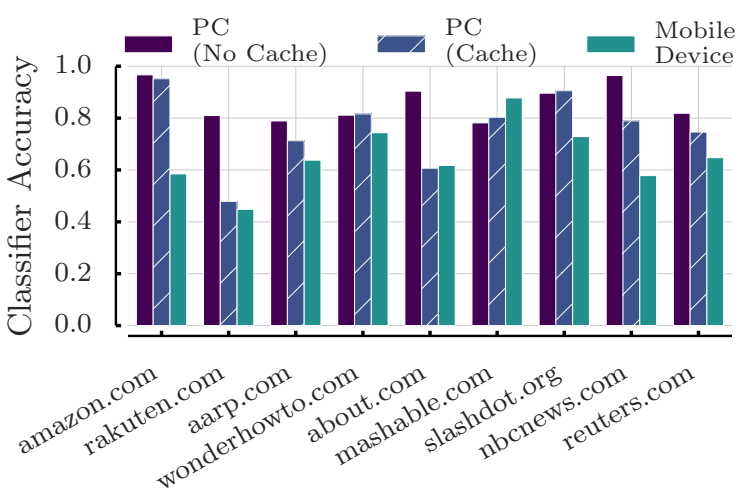

Figure 2: Accuracy of the classifier when fetching pages from a PC (with and without cache) and from a Mobile Device.

any page outside of the set monitored by the eavesdropper. We show that webpage fingerprinting can be reasonably accurate in a closed-world scenario in which the eavesdropper monitors all the pages that the client can possibly visit. This assumption is realistic in our settings because the eavesdropper knows the website requested by the user (by looking at the SNI in the client_hello message) and must infer which page she is browsing within that website.

The features we extract from the traffic generated by downloading a page include: the number of incoming packets and the number of outgoing ones, the total size of incoming packets and the total size of outgoing ones, and a trace defined over the size and the order of the observed packets ${ }^{3}$ We use an SVM classifier with an RBF kernel with $\gamma, c \in[0.001,10000]$. For each website, we capture with tcpdump the traffic generated by fetching each of the 1-st level pages 50 times and measure the accuracy of the classifier using 10 -fold cross validation.

\section{Classifier Accuracy.}

For this experiment we pick 9 websites that have low Jaccard index between the main page and the 1-st

\footnotetext{
${ }^{3}$ Given the space constraints, we refer the reader to 11 for the details on the feature selection process.
} 


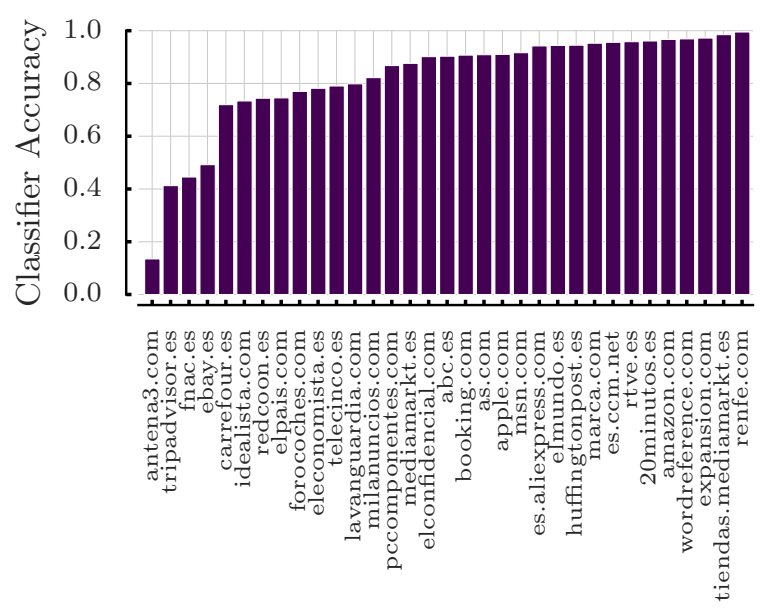

Figure 3: Accuracy of the classifier for the most popular pages in Spain.

level pages (see Figure 1). For each website we train the classifier and test its accuracy in three different scenarios. We use a PC with Mozilla Firefox with and without cache, and a mobile device with Google Chrome with cache enabled. In the latter scenario we use the Android emulator to fetch the pages from an emulated Nexus 5 using the built-in feature of the emulator to simulate the conditions of a $3 \mathrm{G}$ network.

Figure 2 shows the accuracy of the classifier for the 9 websites in each one of the aforementioned scenarios. We found the lower accuracy for the PC with cache scenario when predicting pages of aarp.com (0.79) while we experienced the highest accuracy for amazon.com (0.97). Caching inevitably hinders the accuracy of the classifier by $10.3 \%$ on average, but the average accuracy never drops below 0.48 . The accuracy decreases because when parts of a page are in the local cache, the traffic trace available to the classifier becomes shorter and, therefore, more likely to be confused with that of another page 4 The mobile phone scenario suffers from a similar issue, not due to caching only, but also because mobile versions of a website are typically simpler than their desktop counterparts, and thus they end up producing more similar traffic traces.

In order to confirm the results of the previous experiment on a larger number of websites, we selected the 100 most visited websites from the traces collected by the main network operator in Spain. After manually filtering search engines (i.e., www.google.com/), websites that require the user to login (i.e., www. facebook.com/) and those ones dedicated to content delivery or advertising (i.e., www.doubleclick.net/), we were left with 33 websites including online news-

\footnotetext{
${ }^{4}$ In the extreme case of a page whose elements are all in the cache, the resulting trace becomes totally indistinguishable from that of any other fully cached page.
}

papers, retailers and other websites such as the main railway company in Spain (www.renfe.com/).

We have trained a classifier for each one of the selected website in the "PC, No-cache" scenario. As shown in Figure 3, profiling accuracy is higher than $90 \%$ for 18 of the 33 websites, and higher than $70 \%$ for 29 of them. We experienced worse results on 4 websites: the profiling accuracy on www.antena3. com/, www.tripadvisor.es/, www.fnac.es/ and www. ebay.es/ was 13, 41, 45 and $49 \%$ respectively. Those websites use a common template to show most of the internal pages and leverage CDNs to deliver content. Since our system does not consider data fetched from other domains (i.e., $\mathrm{CDNs}$ ) the common template of the internal pages make webpage prediction a hard task. We speculate that jointly considering the data fetched from CDNs will increase the profiling accuracy but defer this to future work.

\section{From Page Prediction to User Profiling.}

In this experiment we take a closer look at the effect of the classifier accuracy on the quality of the user profiles built by the eavesdropper.

Figure 4a shows the confusion matrix for edition. cnn.com/ where pages are sorted lexicographically based on their URL. For the same website and the same sorting of its pages, the matrix in Figure $4 \mathrm{~b}$ shows the Jaccard index between any pair of 1stlevel pages. Due to the sorting, pages under the same branch of the website, say edition.cnn.com/style/appear sequentially, in both matrices. Figure 4a shows that when the classifier makes a mistake, the output page tends to be "close" to the correct one. For example edition.cnn.com/style/arts/is often mis-classified as edition.cnn.com/style/fashion/and viceversa. This is because the features we use to train the classifier look at the structure of a page (e.g., the number and position of textboxes) rather than its content (e.g., the actual text). Therefore, when pages within the same branch of a website share a similar structure, we experience classification mistakes similar to the ones of Figure 4a.

When mis-classification happens, the amount of damage to user profiling accuracy depends on whether the categories of the true page and the categories of the page output by the classifier overlap or not. For example, because of their similar structure, edition. cnn.com/asia/is likely to be predicted as edition.cnn. com/africa/ by the classifier (see box 1 in Figure 4a); however, given that the set of their categories is very similar (see box 1 in Figure $4 \mathrm{~b}$, the mistake of the classifier has very little impact on the quality of user profiling. Of course this is not always the case. For example the pages under edition.cnn.com/style/ (see box 2 in Figure 4a are likely to be confused with one an other by the classifier. This, however, leads to high profiling error because different pages under 


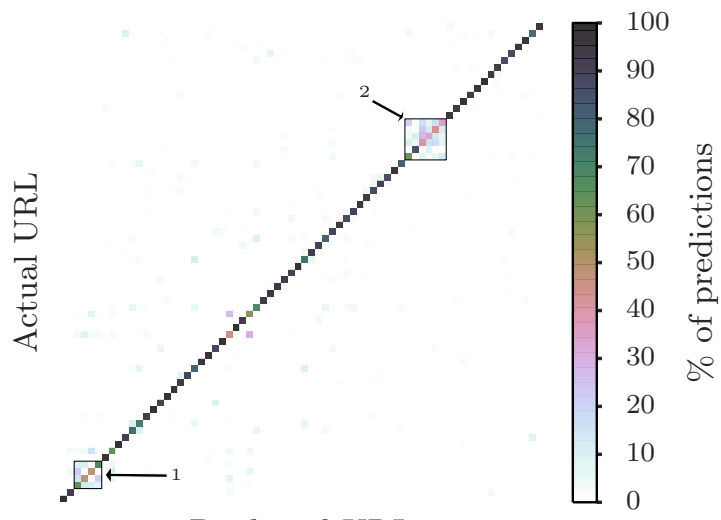

Predicted URL

(a)

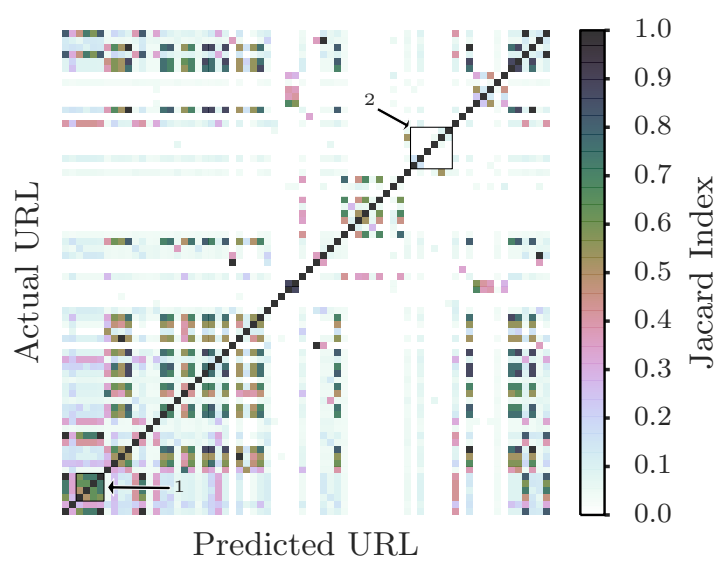

(b)

Figure 4: Confussion matrix (a) of the classifier and the Jaccard index between the categories assigned to two different pages (b) for edition.cnn.com. Box number 1 highlights URLs of the type edition.cnn.com/[region]. Box number 2 shows URLs under the branch edition.cnn.com/style/

the "style" branch of the website have little overlap in term of categories (see box 2 in Figure $4 \mathrm{~b}$ ).

Figure 5 depicts the performance of the basic and the advanced profiling techniques when monitoring the 9 websites of Figure 2. Dashed bars show the precision and recall of the basic profiling technique described in Section 3. Solid bars show the precision and recall of the advanced profiling mechanism that leverages the web fingerprinting technique described above. User profiling leveraging web fingerprinting clearly outperforms the basic profiling technique.

\section{Classifier Freshness.}

The difference between the time when the classifier is trained and the time when pages are predicted may affect the prediction accuracy. This is especially true for very dynamic websites (e.g., news or online community websites). In this experiment we discretize

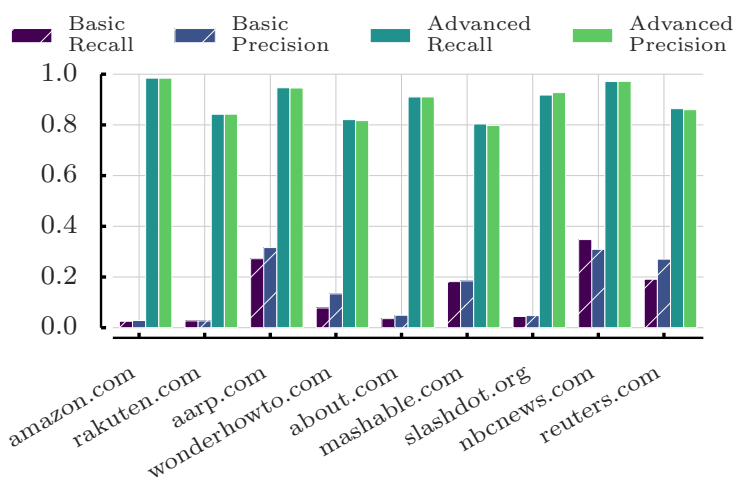

Figure 5: Precision and recall of the baseline eavesdropper and the eavesdropper leveraging website fingerprinting.

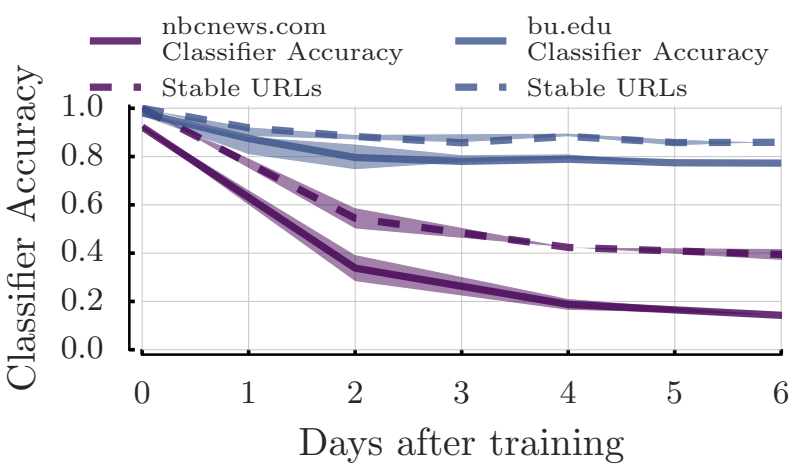

Figure 6: Accuracy of the classifier days after training for a dynamic website (nbcnews.com) and a static one (bu.edu).

time in epochs and we assume that website content only change from one epoch to the next one. If the train and the test data are collected in the same epoch, we say that the classifier is fresh; otherwise we say that the classifier is stale. We define epochs as days. We train the classifier over a snapshot of the website on a given day, and we try to predict pages fetched throughout the following 6 days.

We expect a detectable difference in accuracy between a stale classifier and a fresh one for dynamic pages where content changes every day (e.g., news websites). In the case of websites with static content, the difference between a stale classifier and a fresh one are expected to be less pronounced. To verify this, we add 4 websites with mostly static content (2 corporate and 2 academic ones) to the the 9 websites of the previous experiments.

Figure 6 shows the effect of staleness on the accuracy of the classifier for both a static and a dynamic website. The dashed lines represent the percentage of 1st-level pages that remain linked in the main page across days, while the solid ones represent the accuracy of the classifier. We observe the accuracy of the classifier for the dynamic website decreases rapidly 
while the accuracy for the static one decreases slowly during the first two days and then stabilizes around $80 \%$ accuracy. For both lines, the shadows denote the minimum and the maximum of the statistics.

\section{OPTIMIZING BANDWIDTH USE}

In a real-world deployment, the eavesdropper may not have the bandwidth required to refresh the classifier of each monitored website at every epoch. In the following we formulate an optimization problem for maximizing the profiling quality given a bandwidth constraint.

We consider an eavesdropper that monitors a corpus of $n$ websites $w^{1}, \ldots, w^{n}$. Website $w^{i}$ has a main page $p_{0}^{i}$ and $s^{i} 1$-st level pages $p_{1}^{i}, \ldots, p_{s_{i}}^{i}$. We also use $c\left(p_{j}^{i}\right)$ to denote the set of categories of page $p_{j}^{i}$. When browsing website $w^{i}$, the user may visit any page $p_{j}^{i}$, with $j=0, \ldots, s_{i}$. Since the connection is encrypted, we do not make any assumption on which are the most popular pages within $w^{i}$. If the user visits page $p_{j}^{i}$, the correct categories that should be assigned to that user when browsing $w^{i}$ are, therefore, $c\left(p_{j}^{i}\right)$. We consider any category in $c\left(p_{j}^{i}\right)$ that the profiler assigns to that user as a true positive. Similarly, any category not in $c\left(p_{j}^{i}\right)$ that the profiler assigns to that user is a false positive.

The basic eavesdropper of Section 3 learns $w^{i}$ from the client_hello message issued by the user browser and assigns the categories of the main page $c\left(p_{0}^{i}\right)$ to that user. However, because of HTTPS, the baseline profiling system cannot tell which page $p_{j}^{i}$ was visited. If we denoted with $T^{i}$ and $F^{i}$ the true positive and the false positive, respectively, we have:

$$
\begin{aligned}
& \text { - } T^{i}=\frac{1}{s_{i}+1} \sum_{j=0 . . s_{i}}\left|c\left(p_{0}^{i}\right) \cap c\left(p_{j}^{i}\right)\right| \\
& \text { - } F^{i}=\frac{1}{s_{i}} \sum_{j=1 . . s_{i}}\left|c\left(p_{j}\right) \backslash c\left(p_{0}\right)\right|
\end{aligned}
$$

The advanced eavesdropper of Section 4 tries to infer the page $p_{j}^{i}$ the user has fetched by looking at the encrypted traffic trace. This is done by means of a classifier trained on a snapshot of $w^{i}$. As shown in the previous section, the freshness of the snapshot used to train the classifier impacts its accuracy.

We denote the expected number of true positives and false positives with a classifier that is $t_{i}$ epochs stale by $\mathcal{T}_{t_{i}}^{i}$ and $\mathcal{F}_{t_{i}}^{i}$, respectively. Thus we have:

$$
\begin{aligned}
\text { - } & \mathcal{T}_{t_{i}}^{i}=\sum_{j=0 . . s_{i}} \pi\left(p_{j}^{i}, p_{j}^{i}\right)\left|c\left(p_{j}^{i}\right)\right|+ \\
& \sum_{j=0 . . s_{i}} \sum_{l=0 . . s_{i}, l \neq j} \pi\left(p_{j}^{i}, p_{l}^{i}\right)\left|\left(c\left(p_{j}^{i}\right) \cap c\left(p_{l}^{i}\right)\right)\right| \\
\text { - } & \mathcal{F}_{t_{i}}^{i}=\sum_{j=0 . . s_{i}} \sum_{l=0 . . s_{i}, l \neq j} \pi\left(p_{j}^{i}, p_{l}^{i}\right)\left|c\left(p_{l}^{i}\right) \backslash c\left(p_{j}^{i}\right)\right|,
\end{aligned}
$$

where $\pi\left(p_{j}^{i}, p_{l}^{i}\right)$ denotes the probability of predicting page $p_{j}^{i}$ as $p_{l}^{i}$ (depends on the freshness of the classifier).
Given the expected number of true and false positives, we set $B$ as the bandwidth budget made available to eavesdropper at every epoch, and $b^{i}$ as the bandwidth required to refresh the classifier for webiste $w^{i}$. We also denote by $u^{i}$ the popularity of website $w^{i}$ (i.e., the number of users that visit $w^{i}$ in an epoch).

Upon every epoch, the eavesdropper decides to spend the budget $B$ by training classifiers on a fresh snapshots of a subset $X$ of the monitored websites. If website $w^{i}$ is included in $X$, the available budget is reduces by $b^{i}$ and the expected number of correct categories assigned is $u^{i} \cdot \mathcal{T}_{0}^{i}$, while the expected number of categories miss-assigned is $u^{i} \cdot \mathcal{F}_{0}^{i}$. If website $w^{i}$ is not included in $X$, the budget remains untouched and the expected number of correctly assigned and mis-assigned categories is $u_{i} \cdot \mathcal{T}_{t_{i}}^{i}$ and $u^{i} \cdot \mathcal{F}_{t_{i}}^{i}$, respectively, assuming the most recent classifier for $w^{i}$ is $t_{i}$ epochs stale.

The selection of $X$, therefore, tries to maximize the number of true positive and to minimize the number of false negative, while respecting the available budget.

$$
\begin{aligned}
& \text { Select } X \subseteq\{1, \ldots, n\} \\
& \text { s.t. } \operatorname{Max} \sum_{i \in X} u^{i}\left(\mathcal{T}_{0}^{i}-\mathcal{F}_{0}^{i}\right)+\sum_{i \notin X} u_{i}\left(\mathcal{T}_{t_{i}}^{i}-\mathcal{F}_{t_{i}}^{i}\right) \\
& \text { Where } \sum_{i \in X} b^{i} \leq B
\end{aligned}
$$

If a classifier were never trained on $w^{i}$ we fall-back to the profiling technique of the naïve eavesdropper so that the number of true positives is $u^{i} \cdot c\left(p_{0}^{i}\right)$ and the number of false positive is $\left|c\left(p_{j}^{i}\right) \backslash c\left(p_{0}^{i}\right)\right|$.

The above problem resembles the well-known 0/1 knapsack problem with the only difference that items that are not selected add a non-zero value to the total gain.

\section{A toy example.}

To illustrate the workings and the value of the above optimization we have conducted a simulation based on the 15 websites from previous sections. We empirically assessed the training bandwidth requirement and probabilities of the confusion matrices, while we used Alexa to obtain the popularity of each website.

In Figure 7 we use a black box to mark a domain that is being selected for re-training on a particular day. We show which domains get to be classified every day under two different budgets - $500 \mathrm{mb}$ and $2 \mathrm{~Gb}$, representing $10 \%$ and $40 \%$, respectively of the budget needed to re-classify all sites every day.

We observe that bandwidth availability can strongly affect the daily classification pattern. In case of $500 \mathrm{mb}$ budget, the same set of websites gets to be picked for 


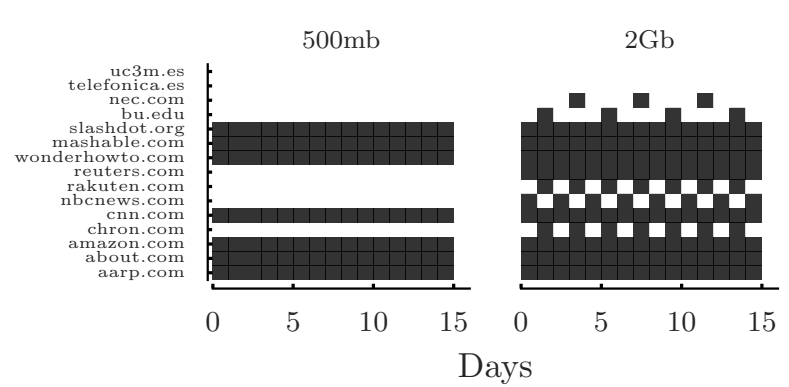

Figure 7: Output of the optimization problem across 15 days for 2 different bandwidth budgets $(500 \mathrm{mb}$ and $2 \mathrm{~Gb}$ ). A black box represents a website which classifier must be refreshed on that day.

classification every day. In the case of $2 \mathrm{~Gb}$, however, different websites get to compete for the available budget and thus end up being picked or skipped on different days. The actual resulting pattern depends on the interplay between website popularity, size, and dynamicity of content.

The small number of websites in the above example does not leave a lot of margin for profiling performance difference between optimizing only once vs optimizing every day. We have, however, simulated a larger example that includes 200 pages with a mix of popularities, content dynamicity, and size and have observed that in more complex settings the difference between optimizing only once vs. every day is substantial.

\section{CONCLUSIONS}

To the best of our knowledge our study is the first one to demonstrate that network eavesdroppers can profile user interests despite HTTPS. We have shown that even off-the-shelf traffic classification algorithms can guess the page that a user is viewing. Caching and dynamic content tailored to the device capabilities make the whole effort harder but the obtained accuracy remains high. We believe that more specialized classification algorithms, coupled with careful optimisation of classification bandwidth can yield accurate and scalable user profiling even in more complex settings than the ones we have considered. We plan to prove our claim by developing a fully functioning prototype.

\section{Acknowledgments}

We are grateful to Juan Miguel Carrascosa, Ruben Cuevas and Costas Iordanou for helpful comments and discussions. We also thank anonymous reviewers and our shepherd for their valuable comments and suggestions. This work has been partially supported by the European Union through the FP7 METRICS (607728), H2020 TYPES (653449) and ReCRED(653417) Projects.

\section{REFERENCES}

[1] J. M. Carrascosa, J. Mikians, R. Cuevas, V. Erramilli, and N. Laoutaris, "I always feel like somebody's watching me measuring online behavioural advertising," in Proc. of $A C M$ CoNEXT'15.

[2] "Display Planner basics." https://support. google.com/adwords/answer/3056115?hl=en. "[Online; accessed 12-May-2016]".

[3] "SSL compliance." https://support.google.com/ richmedia/answer/6015286?hl=en. "[Online; accessed 12-May-2016]".

[4] M. Belshe, R. Peon, and M. Thomson, "Hypertext transfer protocol version 2 (http/2)," RFC 7540, RFC Editor, May 2015. http://www.rfc-editor.org/rfc/rfc7540.txt

[5] "HTTPS Everywhere." https://addons.mozilla. org/en-US/firefox/addon/https-everywhere/ "[Online; accessed 12-May-2016]".

[6] R. Dingledine, N. Mathewson, and P. Syverson, "Tor: The second-generation onion router," tech. rep., DTIC Document, 2004.

[7] T.-F. Yen, Y. Xie, F. Yu, R. P. Yu, and M. Abadi, "Host fingerprinting and tracking on the web: Privacy and security implications.," in Proc. of NDSS'12.

[8] A. Hintz, "Fingerprinting websites using traffic analysis," in Proc. of PETS'02.

[9] D. Herrmann, R. Wendolsky, and H. Federrath, "Website fingerprinting: attacking popular privacy enhancing technologies with the multinomial naïve-bayes classifier," in Proc. of CCSW'09.

[10] A. Panchenko, L. Niessen, A. Zinnen, and T. Engel, "Website fingerprinting in onion routing based anonymization networks," in Proc. of ACM WPES'11.

[11] A. Panchenko, F. Lanze, A. Zinnen, M. Henze, J. Pennekamp, K. Wehrle, and T. Engel, "Website fingerprinting at internet scale," in Proc. of NDSS'16.

[12] "Alexa Top Sites." http://www.alexa.com/topsites. "[Online; accessed 12-May-2016]". 